\title{
Glossectomia como alternativa à recidiva de maloclusão: relato de caso
}

Glossectomy as an alternative to malocclusion recurrence: case report

Glosectomía como alternativa a la recidiva de maloclusión: relato de caso Vítor Bruno TESLENCO ${ }^{1}$

Maylson Alves Nogueira BARROS ${ }^{2}$

Guilherme Nucci dos REIS ${ }^{3}$

Herbert de Abreu CAVALCANTI ${ }^{4}$

\begin{abstract}
Residente em Cirurgia e Traumatologia Bucomaxilofacial, Associação Beneficente da Santa Casa, 79002-251, Campo Grande - MS
${ }^{2}$ Residente em Cirurgia e Traumatologia Bucomaxilofacial, Associação Beneficente da Santa Casa, 79002-251, Campo Grande-MS

${ }^{3}$ Preceptor do programa de residência em Cirurgia e Traumatologia Bucomaxilofacial, Associação Beneficente da Santa Casa, 79002-251, Campo Grande - MS

Coordenador do programa de residencia em Cirurgia e Traumatologia Bucomaxilofacial, Associação Beneficente da Santa Casa, 79002-251, Campo Grande - MS
\end{abstract}

\section{Resumo}

Introdução: a macroglossia é uma condição incomum e pode ser caracterizada como uma desordem por hipertrofia muscular, podendo ter também origem tumoral, endócrina, causas congênitas e doenças adquiridas. Inúmeras são as técnicas cirúrgicas para correção desta anomalia, porém, o plano de tratamento deve ter como base a etiologia da macroglossia. A correção cirúrgica objetiva a retomada de forma e função da língua, reestabelecendo a capacidade mastigatória, respiratória, fonética e estabilidade oclusal. Objetivo: relatar a comunidade científica um caso de glossectomia parcial para correção de um quadro de macroglossia, facilitando assim, a estabilidade do tratamento ortodôntico do paciente. Relato de caso: Paciente de 50 anos, leucoderma, em tratamento por ortodontia corretiva há mais de 18 meses, sem sucesso observado. Devido ao quadro de instablidade ortodôntica, maloclusão sem resolução passiva, macroglossia diagnosticada e redução da capacidade respiratória foi optado por realizar a glossectomia parcial. O procedimento foi realizado sob anestesia geral, onde removemos o tecido muscular a partir da abordagem de buraco de fechadura (Técnica de Kole). Conclusão: constatamos no presente caso, que a técnica de buraco de fechadura empregada neste paciente se mostrou eficaz, uma vez que obtivemos uma melhora estética e funcional, diminuindo o comprimento e largura da língua. Da mesma maneira, o tratamento ortodôntico foi passível de ser finalizado.

Descritores: Macroglossia; Cirurgia Bucal; Glossectomia.

\section{Abstract}

Introduction: Macroglossia is an uncommon condition and can be characterized as a disorder due to muscular hypertrophy, and may also have tumor, endocrine, congenital causes and acquired diseases. There are many surgical techniques to correct this anomaly, however, the treatment plan should be based on the etiology of the macroglossia. Surgical correction aims to resume form and function of the tongue, reestablishing the masticatory, respiratory and phonetic capacity of the patient. Objective: to report to the scientific community a case of glossectomy for correction of a macroglossia, thus facilitating the stability of the patient's orthodontic treatment. Case report: Fifty-year-old patient, leucoderma, undergoing corrective orthodontic treatment for more than 18 months, with no observed success. Due to the orthodontic instability, malocclusion without passive resolution, diagnosed macroglossia and reduction of respiratory capacity, we opted to perform a partial glossectomy. The procedure was performed under general anesthesia, where we removed the muscle tissue from the keyhole approach (Kole Technic). Conclusion: in the present case, the locking technique used in this patient proved to be effective, since we obtained an aesthetic and functional improvement, reducing the length and width of the tongue. In the same way, the orthodontic treatment was able to be finalized.

Descriptors: Macroglossia, Surgery, Oral; Glossectomy.

\section{Resumen}

Introduccíon: La macroglossia es una condición inusual y puede ser caracterizada como un desorden por hipertrofia muscular, pudiendo tener también origen tumoral, endocrina, causas congénitas y enfermedades adquiridas. En numerosas son las técnicas quirúrgicas para la corrección de esta anomalía, sin embargo, el plan de tratamiento debe basarse en la etiología de la macroglossia. La corrección quirúrgica objetiva la reanudación de forma y función de la lengua, restableciendo la capacidad mastita, respiratoria y fonética del paciente. Objetivo: relatar la comunidad científica un caso de glossectomía para la corrección de un cuadro de macroglossia, facilitando así la estabilidad del tratamiento ortodóntico del paciente. Relato de caso: Paciente de 50 años, leucoderma, en tratamiento por ortodoncia correctiva desde hace más de 18 meses, sin éxito observado. Debido al cuadro de instablidad ortodóntica, maloclusión sin resolución pasiva, macroglossia diagnosticada y reducción de la capacidad respiratoria fue optado por realizar la glossectomía parcial. El procedimiento fue realizado bajo anestesia general, donde removemos el tejido muscular a partir del abordaje de agujero de cerradura (Técnica de Kole). Conclusión: constatamos en el presente caso, que la técnica de agujero de cerradura empleada en este paciente se mostró eficaz, una vez que obtuvimos una mejora estética y funcional, disminuyendo la longitud y anchura de la lengua. De la misma manera, el tratamiento ortodóntico fue pasible de ser finalizado.

Descriptores: Macroglosía; Cirugía Bucal; Glosectomía.

\section{INTRODUÇÃO}

A macroglossia é uma condição incomum e pode ser caracterizada como uma desordem por hipertrofia muscular, podendo ter também origem tumoral, endócrina, causas congênitas e doenças adquiridas $^{1,2}$. Casos de hipertrofia da língua já foram associados até mesmo a amiloidose ${ }^{3}$. Por muitos anos, a correção cirúrgica desta patologia foi tida como última opção, porém, nos dias atuais sabe-se que é o melhor plano de tratamento para a melhora da qualidade de vida do paciente ${ }^{1,4}$.

O paciente portador desta deformidade pode apresentar diversas alterações, tais como: mal oclusão dentária, má formação dos músculos da mastigação e ossos gnáticos, perda da função mastigatória, dificuldades respiratórias, problemas de fonação e instabilidade no tratamento ortodôntico ${ }^{5}$. Deve-se ter muito cuidado no momento do diagnóstico, uma vez que o cirurgião pode estar se deparando com uma condição denominada pseudomacroglossia, onde o paciente apresenta um tamanho normal da língua, porém apresenta uma atrofia das estruturas gnáticas adjacentes ${ }^{1}$.

Inúmeras são as técnicas cirúrgicas para correção desta anomalia, porém, o plano de tratamento deve ter como base a etiologia da macroglossia, ${ }^{1,6}$ A correção cirúrgica objetiva a retomada de forma e função da língua, reestabelecendo a capacidade mastigatória, 
respiratória e fonética do paciente ${ }^{1,3,6}$. As técnicas variam em seu formato de incisão, porém a maioria tem como resultado a diminuição da extensão lingual em largura e comprimento ${ }^{6}$.

Dentre as técnicas de excisão periférica podemos citar a de Butlin, Ensin, Harris, Blair, Hendrick, Edgerton, Dingman, Grabb e Gupta tinham como objetivo reduzir o comprimento e a largura da língua. Com o passar dos anos, surgiram variáveis destar técnicas primordiais, como a de Krunchinsky, Mixter, e Harda que foram projetados para preservar a ponta da língua, uma área vital para a sensação de gosto e fala6. Nos dias atuais, uma das técnicas mais difundidas e utilizadas é a de buraco de fechadura de Kole $^{6,7}$.

Desta maneira, o presenta trabalho tem como proposição relatar um caso de instabilidade no tratamento ortodôntico por macroglossia, onde se fez necessária correção cirúrgica.

\section{CASO CLÍNICO}

Paciente de 50 anos, leucoderma, hipertenso e diabético controlado, em tratatamento por ortodontia corretiva há mais de 18 meses, sem sucesso observado. Devido ao quadro de instabilidade ortodôntica, maloclusão sem resolução passiva (o que impossibilitada preparo ortocirurgico para ortognática), macroglossia diagnosticada e redução da capacidade respiratória (programação de correção por meio de cirurgia ortognática), o paciente foi encaminhado pelo ortodontista e fonoaudióloga à equipe de Cirurgia Bucomaxilofacial para avaliação e realização de glossectomia parcial.

Foi confirmado então, quadro de macroglossia, onde foi indicada redução de parte da musculatura lingual, a fim de se aumentar espaço de via aérea, melhorar a capacidade mastigatória e possibilitar o término do preparo ortocirúrgico. (Figura 1).

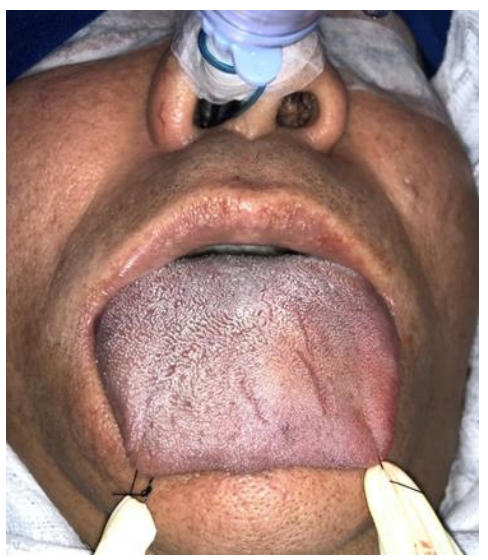

Figura 1: Pré-operatório, evidenciando o aumento do tecido muscular.

O mesmo foi submetido avaliação précirurgica (risco cirurgico) pelo cardiologista. Por ser portador de hipertensão arterial sistêmica, dislipidemia e diabetes melitus tipo II, em uso de losartana 50m, glifage e lipless, respectivamente. Por apresentar normalidade em todos exames laboratoriais, eletrocardiograma e radiografia de tórax, o mesmo foi classificado como ASA II / Goldman I, estando apto para que fosse realizado procedimento cirúrgico. Seguimos então para realização do procedimento, sob anestesia geral, entubação nasotraqueal. Devido a necessidade de remoção apenas em comprimento e largura, optamos pela técnica de Kole, uma vez que a quantidade de retirada de tecido muscular era extensa.

Iniciamos o procedimento com a demarcação das linhas de incisão, utilizando azul de metileno (Figura 2).

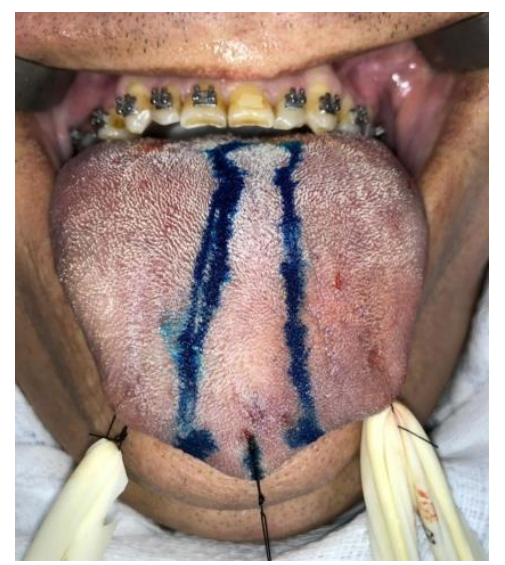

Figura 2: Demarcação da incisão, utilizando azul de metileno.

Em sequência, foi realizada infiltração em toda região que foi abordada, utilizando solução de lidocaína com epinefrina 1:100.000, sob aspiração negativa. Uma incisão superficial com lâmina de bisturi número 15 foi feita no traço demarcado (Figura 3).

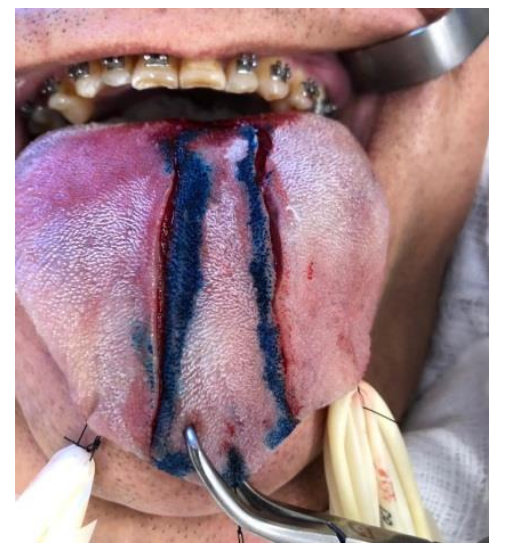

Figura 3. Incisão inicial realizada com lâmina 15 .

Os planos profundos foram incisados utilizando-se eletrodo de bisturi elétrico monopolar, do tipo colorado, realizando-se hemostasia quando necessário por fulguração ou ligando vasos com vicryl 4-0. Após remoção de todo o tecido muscular previsto, realizamos a síntese dos planos internos com ácido poliglicólico número 3-0 e nos planos mais superficiais foi optado pelo mesmo fio, porém em menor diâmetro (4-0). Quando foi observada total hemostasia, o paciente foi liberado, onde o pósoperatório na sala de recuperação anestésica se deu 
sem intercorrências (Figuras 4 a 7).

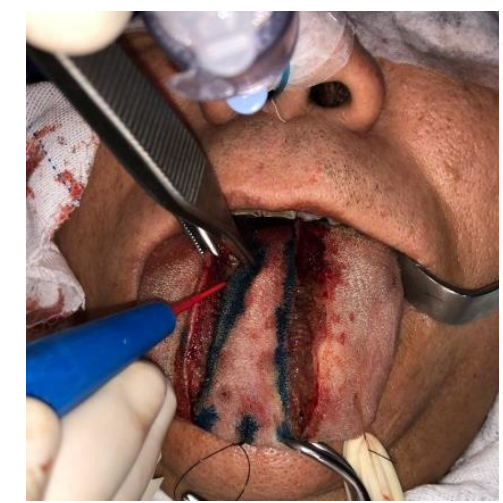

Figura 4. Incisão em tecido muscular profundo, com auxílio de eletrodo tipo colorado.

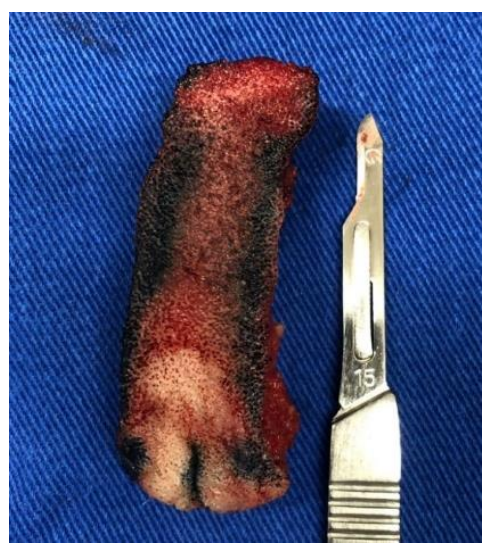

Figura 5. Tecido removido.

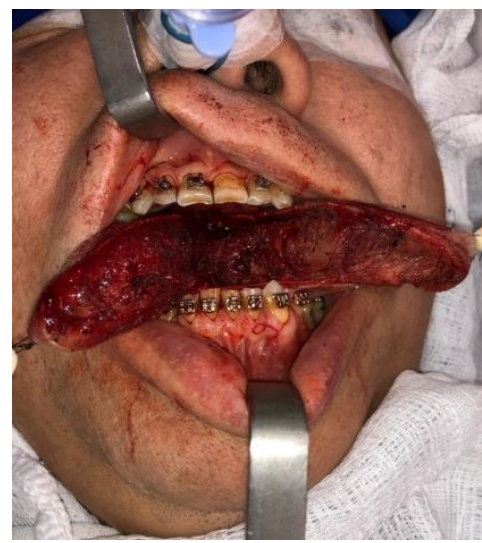

Figura 6. Retalho dividido.

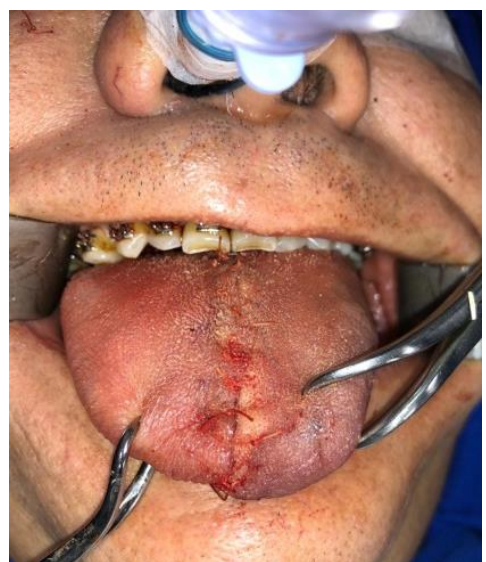

Figura 7. Aspecto final imediato após síntese com ácido poliglicólico 4-0.

Com a total cicatrização do procedimento, o paciente foi então encaminhado ao ortodontista, para continuidade do tratamento ortodôntico. Assim como tratamento pela equipe de fonoaudiologia, uma vez que sua capacidade fonética se mostrou alterada após o procedimento.

\section{DISCUSSÃO}

O diagnóstico do paciente portador de macroglossia nem sempre é fácil, uma vez que a acomodação da língua entre o espaço da cavidade oral pode ser mascarada pelo tratamento ortodôntico ${ }^{1}$. Sua mensuração pode ser feita de maneira direta ou indireta, com auxílio de exames de imagem, como a ressonância magnética ou mensurações cefalométricas ${ }^{1}$.

Após a escolha de tratamento por glossectomia parcial, o paciente deve estar ciente dos riscos acerca do procedimento ${ }^{1,6,8}$. Podemos citar entre eles a perda de sensação gustatória (conforme a técnica selecionada), formação de hematomas, hemorragias trans ou pós-operatórias, obstrução de vias aéreas por edema, alteração da fonação, disfunção motora por lesão neural (hipoglosso) e injúrias ao ducto salivar ${ }^{6-8}$.

Dentre os requisitos para submeter o paciente à uma glossectomia parcial, devemos avaliar o volume da língua, sua mobilidade, fonação do paciente, mordida aberta esqueletal, interferência ou instabilidade no tratamento ortodôntico, babação, deglutição deficiente e trauma lingual decorrente ${ }^{1,6-10}$. No presente estudo, optou-se por esta modalidade de tratamento, devido ao paciente apresentar acometimento estético e recidiva de maloclusão após tratamento ortodôntico.

Podemos citar dentre as técnicas descritas na literatura, as chamadas glossectomias a partir da linha mediana e as periféricas. Em 1965 Kole propôs uma incisão triangular na região do ápice lingual, porém, posteriormente desenvolveu uma modificação de sua própria técnica, estendendo-se ao doso lingual, realizando uma incisão em formato de "buraco de fechadura" ou "keyhole". Sua técnica se difundiu entre os cirurgiões devido a fácil reprodutibilidade e bom resultado estético-funcional ${ }^{6,7,8}$. Porém, em casos onde a necessidade de remoção tecidual é pequena, podemos lançar mão de técnicas periféricas, como a de Butlin and Ensign ${ }^{6}$. No presente caso, optou-se pela abordagem de Kole, pois se mostrou necessária remodelação da língua apenas em comprimento e largura, descartando a necessidade de alteração em altura ${ }^{6}$.

Os pacientes que apresentam a macroglossia e serão submetidos à um procedimento de glossectomia parcial, devem estar cientes da necessidade de acompanhamento fonoaudiólogo no pós-operatório ${ }^{1,3,4,11}$.

O tratamento das macroglossias ainda não é muito bem definido, uma vez que existem inúmeras técnicas para correção cirúrgica. Desta maneira, se mostra como consenso na comunidade científica que 
CONFLITO DE INTERESSES

Os autores declaram não haver conflitos de interesse. caso, a fim se de estabelecer a técnica ideal ${ }^{6}$.

\section{CONSIDERAÇÕES FINAIS}

Constatamos no presente caso, que a técnica de buraco de fechadura empregada neste paciente se mostrou eficaz, uma vez que obtivemos uma melhora estética e funcional, diminuindo o comprimento e largura da língua. Da mesma maneira, o tratamento ortodôntico foi passível de ser finalizado.

\section{REFERÊNCIAS}

1. Topouzelis N, Iliopoulos C, Kolokitha OE. Macroglossia. Int Dent J. 2011;61(2):63-9.

2. Neville BW, Allen CM, Damm DD, Chi AC. Patologia: oral e maxilofacial. 4.ed. Rio de Janeiro: Guanabara Koogan; 2016.

3. Gadiwalla Y, Burnham R, Warfield A, Praveen P. Surgical management ofmacroglossia secondary to amyloidosis. BMJ Case Rep. 2016:10.1136.

4. Salmen FS, Dedivitis RA. Partial glossectomy as an auxiliary method to orthodontic treatment of dentofacial deformity. Int Arch Otorhinolaryngol. 2012;16(3):414-17.

5. Costa SAP, Brinhole MCP, da Silva RA, Dos Santos DH, Tanabe MN. Surgical treatment of congenital true macroglossia. Case Rep Dent. 2013;2013:489194.

6. Balaji SM. Reduction glossectomy for large tongues. Ann Maxillofac Surg. 2013;3(2):167-72.

7. Cymrot M, Teixeira FAA, Sales FCD, Muniz NFJ. Glossectomia subtotal pela técnica de ressecção lingual em orifício de fechadura modificada como tratamento de macroglossia verdadeira. Rev Bras Cir Plást. 2012;27(1):165-69.

8. Tanaka OM, Guariza-Filho O, Carlini JL, Oliveira DD, Pithon MM, Camargo ES.Glossectomy as an adjunct to correct an open-bite malocclusion with shortenedmaxillary central incisor roots. Am J Orthod Dentofacial Orthop. 2013;144(1):130-40.

9. Salmen FS, Dedivitis RA. Glossectomia parcial como método auxiliar ao tratamento ortodôntico da deformidade dento-facial. Int Arch Otorhinolaryngol. 2012;16(3):414-17.

10.Jung YW, On SW, Chung KR, Song SI. Simultaneous glossectomy with orthognathic surgery for mandibular prognathism. Maxillofac Plast Reconstr Surg. 2014;36(5):214-18.

11.Vieira CA. Fonoterapia em glossectomia total: estudo de caso. Rev Soc Bras Fonoaudiol. 2011;16(4):479-82.
Submetido em 19/03/2019

Aceito em 11/06/2019

\section{AUTOR PARA CORRESPONDENCIA}

Vitor Bruno Teslenco

v.teslenco@live.com 